\title{
Immunoanalysis for environmental monitoring and human health
}

\author{
Shirley J. Gee ${ }^{1}$ Ivan R. Kennedy ${ }^{2}$ - Alice Lee $^{3} \cdot$ Hideo Ohkawa ${ }^{4}$. \\ Tippawan Prapamontol ${ }^{5} \cdot$ Ting $\mathrm{Xu}^{6}$
}

Published online: 9 June 2016

(C) Springer-Verlag Berlin Heidelberg 2016

In the 1950s Yalow and Berson developed a radioimmunoassay for insulin. It was the first case of immunoassay used for diagnostics, measuring glucose levels in diabetic patients. Later, Rosalyn Yalow received a Nobel Prize for this effort. In the 1970s Van Weeman and Schuurs replaced the radiolabel with

Published in the topical collection Immunoanalysis for Environmental Monitoring and Human Health with guest editors Shirley J. Gee, Ivan R. Kennedy, Alice Lee, Hideo Ohkawa, Tippawan Prapamontol, and Ting Xu.

\section{Shirley J. Gee}

sjgee@ucdavis.edu

\section{Ivan R. Kennedy}

ivan.kennedy@sydney.edu.au

Alice Lee

alice.lee@unsw.edu.au

Tippawan Prapamontol

tippawan@rihes.cmu.ac.th

Ting Xu

xuting@cau.edu.cn

1 Department of Entomology and Nematology, University of California, One Shields Avenue, Davis, CA 95616, USA

2 Agricultural and Environmental Chemistry, University of Sydney, Sydney, NSW 2006, Australia

3 Food Science and Technology, University of New South Wales, Sydney 2051, Australia

4 Kobe University, Kashiodai 14-14, Kitaku, Kobe 651-1255, Japan

5 Research Institute for Health Sciences, Chiang Mai University, P.O. Box 80 C.M.U., Chiang Mai 50202, Thailand

6 College of Resources and Environmental Sciences, China Agricultural University, No. 2 Yuan Ming Yuan West Road, Haidian District, Beijing 100193, China enzyme labels. This reduced the hazard from radiation, and the turnover of substrate by the enzyme resulted in an amplification of the signal. With radioimmunoassay (RIA) a single binding event was detected by a single radioactive molecule, whereas a single binding event by an enzyme-labeled ligand resulted in increasing color formation as the enzyme turns over substrate. This conversion to enzyme labels also allowed more laboratories to utilize the method since the use of radiolabels often excluded laboratories because of regulatory barriers. Enzyme-linked immunosorbent assays (ELISAs) were also reported to have faster reaction times, greater specificity for the target molecule, and longer shelf lives compared to RIAs.

Accompanying improvements to labels, efforts continue to improve the primary component of the immunoassay, the antibody. Polyclonal antibodies derived from immunizing mammals, primarily rabbits, sheep, goats, and donkey, are a standard reagent. However, the quality of the antibody may not be consistent because of animal to animal variability. In the 1980s, Köhler and Milstein developed a technique that fused antibody-producing B cells to a myeloma cell line. After fusion, an immortal monoclonal cell line producing a single antibody of interest is discovered though screening and results in a cell line that can continuously produce a well-defined reagent. This opened additional doors to commercialization of immunoassays. Recombinant technology has led to the development of antibody molecules expressed in E. coli and the discovery that fragments of the whole antibody molecule retain binding characteristics. Camelid antibodies have been found that have a different morphology consisting of a similar constant region, but the binding portion consists of only the variable heavy chain. This binding portion is $1 / 10$ th the size of the classical antibody while retaining high binding affinity and additional properties of thermal and chaotropic stability. Their small size makes them easier to clone and express and modify 
genetically as well as apply genetic tools to create hybrid antibody-label molecules, such as antibody-alkaline phosphatase fusions. Their easy and high-yield expression makes it more feasible for antibodies to be used in high volume applications such as immunoaffinity and is an obvious plus for commercial applications.

Compared to instrumental methods such as GC-MS or LC-MS, immunoassays have similar sensitivity, are as selective, but more rapid and cost-effective, manage large sample loads well, and are adaptable to analytes that vary in size and complexity. Because of these advantages, immunoassay technology has been applied to a wide variety of analytes. In the 1980s Hammock and Mumma proposed that immunoassays could be used for the analysis of environmental contaminants. Since then immunoassays have been developed for many small molecule environmental contaminants, and the technology has been validated by the Association of Official Analytical Chemists and published as official methods by the Environmental Protection Agency.

Despite the long history of immunoassay analysis, research continues today proposing new uses, unique labels, faster homogeneous formats, novel binding reagents, and multiplexing approaches. The versatility of immunoassays is illustrated by the contributions to this special collection of papers. It is clear that they excel as surveillance methods. For example, several papers describe immunoassays that are intended to protect human health by ensuring that food is not contaminated with chemicals, naturally occurring toxins, pathogens, or allergen residues. Another use is for assessing the quality and integrity of the composition of foods, specialty chemicals, and natural products used or consumed by humans. Immunoassay continues to be an important method in disease diagnostics for both humans and food crops. Especially important is the move to pointof-use detection, including using automatic techniques such as lateral flow devices (LFDs). LFDs require little or no laboratory expertise. Rapid and timely detection of toxic chemicals will minimize environmental problems and reduce human exposure. Similarly early detection of pathogens in food crops will result in minimizing pesticide use and decrease crop losses, while bedside detection of infectious diseases will result in more rapid diagnosis and treatment delivered in a more personalized manner leading to decreased mortality and morbidity.

We anticipate that immunoassay technology will continue to play a large role in the protection of human, animal, and environmental health. Adaptation of the technology to field-portable, point-of-use devices will improve health and safety in both developed and more resource-poor developing countries.

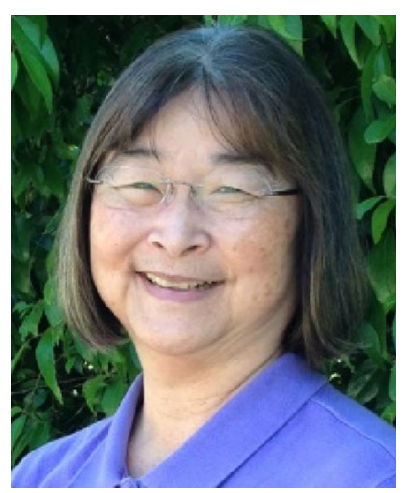

Shirley J. Gee is a Staff Research Associate in the Department of Entomology at the University of California Davis. She received her Master's Degree in Pharmacology and Toxicology and for the past 25 years has contributed to research areas in toxicology and analytical chemistry. Her research interests include the development and application of immunoassay technology for environmental analysis as well as for the measurement of biomarkers of exposure to toxicants. Focusing primarily on pesticides and environmental contaminants, her research group, led in conjunction with Dr. Bruce Hammock, has a strong record in new assays, application of nanoparticle labels, and development of novel binding molecules such as phage-displayed peptides and nanobodies.

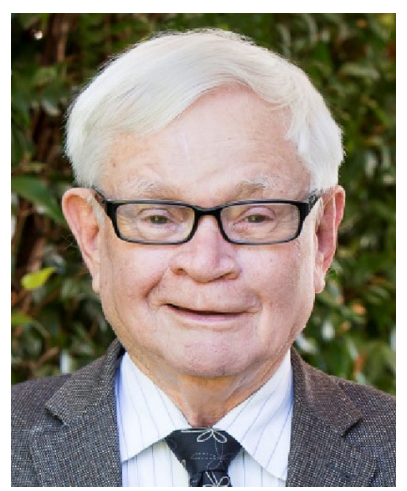

Ivan R. Kennedy is Professor Emeritus in Agricultural and Environmental Chemistry at the University of Sydney and a Fellow of the Royal Australian Chemical Society. With his research students, since the 1970 s he has focused on several areas of applied chemistry and biochemistry regarding production of food of high quality and environmental protection. More recently the research has emphasized risk management based on inexpensive monitoring using ELISA immunoreagents. On retirement from teaching in 2012 he resolved to commercialize his student's research, establishing a company with two former post-docs (Quick Test Technologies) based on using lateral flow devices for quantitative analysis of mycotoxins such as aflatoxin and pesticides in soil and water.

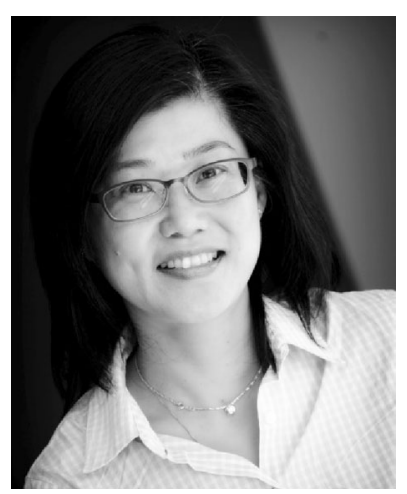

Alice Lee is Associate Professor and Co-Director of the ARC Training Centre for Advanced Technologies in Food Manufacture at the University of New South Wales. Her research program centers around three key integrated areas: analytical chemistry, natural bioactives, and food and environmental safety. In the immunoanalytical area, she has developed immunodiagnostic tests for a wide range of targets including pathogens, pesticides, antibiotics, endocrine disrupting hormones and chemicals, plant alkaloids, and allergens. She combines nanoparticle technology with the immunodiagnostic technology to develop nanoparticle-based immunodiagnostic platforms with enhanced properties. 


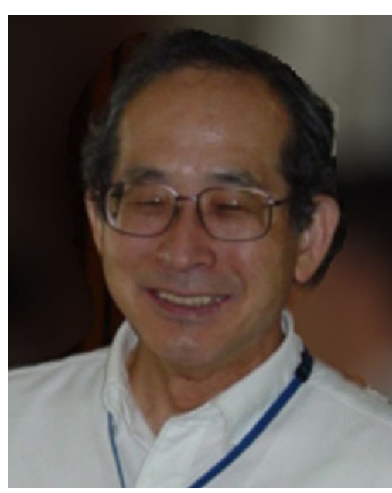

Hideo Ohkawa is Professor Emeritus of the Research Center for Environmental Genomics at Kobe University, Japan, and is an expert in pesticide chemistry having authored more than 300 peer-reviewed publications and edited a number of books. He has a broad interest in agricultural chemistry and the development and utilization of immunoassays for analysis of pesticides and environmental contaminants. $\mathrm{He}$ was instrumental in leading the effort in the application of environmental immunoassay in Japan as the President of the Immunochemical Society of Japan. Having earlier worked in the industrial sector, he has consulted with companies to commercialize the technology and so has a strong interest in the application of standardization of protocols.

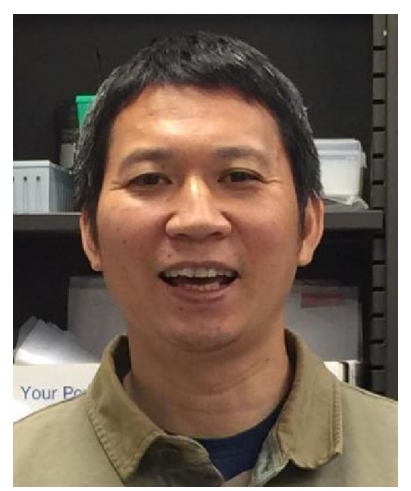

Ting $\mathrm{Xu}$ is a Professor of immunoassay technology in the College of Resources and Environmental Sciences, China Agricultural University, Beijing. His research publications center on the immunoassay method, especially enzyme-linked immunosorbent assays (ELISA), as an effective screening tool for agrochemicals and pollutants in foods and in the environment. His main contributions to science are associated with the design of novel haptens, production of monoclonal and polyclonal antibodies as well as engineered antibodies, and development of competitive and non-competitive ELISAs for small molecules. In addition, application and validation of immunoassays for agrochemicals and pollutants have been extended to food, water, and soil samples.

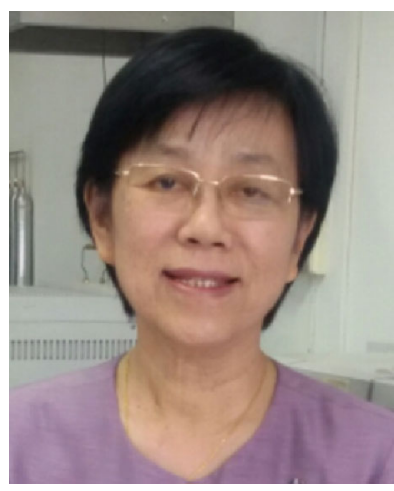

Tippawan Prapamontol is a Senior Researcher at the Environment and Health Research Unit, Research Institute for Health Sciences, and an adjunct lecturer at the Graduate School, Chiang Mai University, Chiang Mai, Thailand. She is a biochemist and environmental toxicologist by training. She served as Head of Center for Pollution and Environmental Health Research, Research Institute for Health Sciences, Chiang Mai University, and currently serves as a co-investigator of an NIH/NIEHS funded study on the relationship of early pesticide exposure and neurodevelopmental outcomes. She recently completed a study, as a lead investigator, on pesticides and air pollutants in upper northern Thailand. 